\title{
Pre-main sequence stars in open clusters
}

\section{The DAY-I catalogue ${ }^{\star}$}

\author{
A. J. Delgado ${ }^{1}$, E. J. Alfaro ${ }^{1}$, and J. L. Yun ${ }^{2}$ \\ 1 Instituto de Astrofísica de Andalucía, CSIC, Apdo 3004, 18080-Granada, Spain \\ e-mail: [delgado;emilio]@iaa.es \\ 2 Centro de Astronomia e Astrofísica da Universidade de Lisboa, Observatório Astronómico de Lisboa, Tapada da Ajuda, \\ 1349-018 Lisboa, Portugal \\ e-mail: joao.yun@oal.ul.pt
}

Received 26 October 2006 / Accepted 30 January 2007

\begin{abstract}
Context. We present the basic ideas and first results from the project we are carrying out at present, the search for and characterisation of pre-main sequence (PMS) stars among the members of Galactic young clusters. The observations of 10 southern clusters, nine of them located in the Carina-Sagittarius spiral arm of the Milky Way are presented.

Aims. We aim at listing candidate PMS member stars in young clusters. The catalogued stars will serve as a basis for future spectroscopic studies of individual objects to determine the properties of stellar formation in the last phases before the main sequence stage. Properties such as the presence of residual envelopes or disks, age spread among PMS members, and the possible presence of several episodes of star formation in the clusters, are to be addressed.

Methods. Multicolour photometry in the $U B V R_{\mathrm{C}} I_{\mathrm{C}}$ system has been obtained for 10 southern young clusters in the fourth Galactic quadrant, located between Galactic longitudes $l=238^{\circ}$ and $l=310^{\circ}$. For six clusters in the sample, the observations presented here provide the first published study based on CCD photometry. A quantitative comparison is performed with post-MS isochrones, and PMS isochrones from three different evolutionary models are used in the photometric membership analysis for possible PMS stars. Results. The observations produce photometric indices in the Johnson-Cousins photometric systems for a total of 26962 stars. The matching of our pixel coordinates with corresponding fields in the 2MASS data base provides astrometric calibration for all cataloged stars and $J H K$ 2MASS photometric indices for $60 \%$ of them. Post-MS cluster ages range from 4 to 60 Myr, whereas the photometric membership analysis assigns PMS membership to a total of 842 stars, covering an age range between 1 and 10 Myr. This information on the PMS candidate members has been collected into a catalogue, named DAY-I, which contains 16 entries for 842 stars in the field of 10 southern clusters.
\end{abstract}

Key words. open clusters and associations - stars: pre-main sequence - catalogs

\section{Introduction}

Stars clusters are commonly accepted as the astrophysical objects best-suited to achieving reliable ages and distances of member stars at any location in the Milky Way. Furthermore, comparing photometric colour-magnitude (CM) diagrams with model isochrones simultaneously provides a way to estimate masses and chemical compositions and to set constraints on the physics of the models themselves. In particular, the systematic observation of the Galactic sample of young clusters offers a number of interesting possibilities for improving knowledge of how the star formation occurs on large scales in the Galaxy (e.g. Alfaro et al. 1991) and how stars form inside the clusters (e.g. Lada 2005; Lada \& Alves 2004). By young clusters we understand objects with ages between 1 and $10 \mathrm{Myr}$, which can be easily and productively observed at both optical and infrared wavelengths and with telescopes around $1 \mathrm{~m}$ in diameter. For objects located not further than 3-4 kpc, we are left with clusters containing observable PMS members, also called Post-T Tauri stars

* Catalog is only available in electronic form at the CDS via anonymous ftp to cdsarc.u-strasbg.fr (130.79.128.5) or via http://cdsweb.u-strasbg.fr/cgi-bin/qcat?]/A+A/467/1397
(Mamajek et al. 2002), with spectral types from A to K, detectable in photometric diagrams deep down to $V$ 21-22, depending on reddening. These observations are feasible with small telescopes and can be obtained for a wide sample of clusters in the nearest complexes of star formation, such as Carina and Cygnus, as well as for some clusters located at greater distances in the direction of the Galactic anticentre.

These observations typically refer to older stars than those located in embedded regions of star formation, such as the Orion nebula. But they still provide invaluable information on the earliest phases of star formation. In particular, they are expected to contain clues to the mass distribution of forming stars in selected regions, and they can also be studied with advantage at optical wavelengths. In this spectral region, the measurement of distance and age by means of ZAMS and post-MS isochrone fittings to the brightest part of the colour-magnitude diagram (CMD) is more reliable and leads to more accurate estimates of physical parameters for the member stars than for isolated objects.

PMS stars in clusters between 1 and 10 million yr are especially interesting for investigating whether O-star formation terminates or, on the contrary, triggers the formation of less massive stars, as well as the possible deficit in the latter case 
(Sung et al. 1998). Furthermore, they trace the evolution through the last phases of circumstellar disk dissipation and angular momentum variation of the central star (Stauffer et al. 1989; Haisch et al. 2001; Slesnick et al. 2002; Sicilia-Aguilar et al. 2005; Hartmann 2005). In particular, the proportion of CTTs to WTTs, possibly as a function of age, can be investigated (Herbig 1998). Recent work reveals the existence of long-lived disks around low-mass PMS stars, favourable for the formation of planetary disks (Lawson et al. 2004; Lyo et al. 2004), and a decrease in the time scale of disk dissipation with increasing mass (Hernández et al. 2005).

The presence of PMS members is addressed in most studies devoted to one or more young clusters, but specific membership assignments are present only for close or very bright objects. Ever since the first systematic investigation by Walker (1961, and references therein) of a group of bright young galactic clusters (NGC 2264, NGC 6530, IC5146, NGC6611), the presence of PMS stars among the members of young clusters has been mentioned frequently in the literature. Many of these studies mention only the location of some stars on photometric diagrams, suggesting their PMS nature (FitzGerald et al. 1978; Forbes \& DuPuy 1978; Sagar \& Joshi 1979, 1981; Stone 1988; Forte \& Orsatti 1984; Witt et al. 1984; Heske \& Wendker 1984; Verschueren et al. 1990; Marschall et al. 1990; Munari et al. 1998; Carraro et al. 2001; Patat \& Carraro 2001).

A few studies include comparison with models and eventually more detailed observations of the most conspicuous PMS candidate members. Their main interest is focused on indications of coevality or non-coevality of the cluster members, the properties of the cluster mass function, and the possible peculiarities of the spatial distribution of the PMS member stars (Stauffer et al. 1989; Trullols \& Jordi 1997; Marco et al. 2001; DeGioia-Eastwood et al. 2001; Marco \& Negueruela 2002). In some cases, membership probability is assigned on the basis of statistical considerations or discussed in some detail based on the global appearance of the CM diagrams as compared to PMS isochrones (Baade 1983; Baume et al. 1999; Baume et al. 2003; Prisinzano et al. 2005)

On the other hand, detailed studies of the PMS candidate members, included in extensive analyses of the parent clusters, have been published mainly for a reduced collection of clusters (Thé et al. 1985; Hillenbrand et al. 1993; de Winter et al. 1997; van den Ancker et al. 1997; Sung et al. 1997; Herbig 1998; Sung et al. 1998; Baume et al. 1999; Delgado et al. 1999; Sung et al. 2000; van den Ancker et al. 2000; Park et al. 2000; Park \& Sung 2002; Delgado et al. 2004, 2006). Some of these studies use previously established PMS members, while others make their own PMS membership assignments, whereby the methods include location in several photometric diagrams, considerations of reddening, spectroscopic information for some stars, and values of specific photometric indices such as $(R-\mathrm{H} \alpha)$.

Most recently, several published works contain exhaustive analyses of the PMS populations in regions close to the Sun, based on the widespread data sets available and including new observations (Mamajek et al. 2002; Luhman et al. 2003; Sartori et al. 2003; Pozzo et al. 2003; Lyo et al. 2004; Prisinzano et al. 2005). For these intensively studied objects, matches with available proper motions catalogues and lists of associated X-ray sources are used, and they provide the best-studied samples of PMS stars. Furthermore, in some special cases - clusters located at close distances, although not necessarily younger than $10 \mathrm{Myr}$ - detailed observations have been recently carried out to compare ages determined from isochrone fits, both for postMS and for PMS models, with ages derived from the theoretical predictions on lithium depletion during the PMS evolution (Jeffries \& Oliveira 2005; Palla et al. 2005).

The conclusions about age spread, mass functions, and differences between evolutionary models differ between studies. There is a general trend to admit the existence of a significant age spread, which also produces age differences as estimated from PMS and post-MS isochrones. Values suggested for age spread among PMS candidate members are roughly 5 Myr (Sung et al. 2000; Park et al. 2000; Park \& Sung 2002), although values from 1 Myr (Hillenbrand et al. 1998) to more than $10 \mathrm{Myr}$ (DeGioia-Eastwood et al. 2001; Pozzo et al. 2003; Prisinzano et al. 2005) are claimed. On the other hand, results from different analyses of the closest associations, using a great wealth of available data offer different conclusions about the age differences between association members; see Mamajek et al. (2002) and Sartori et al. (2003) on the age spread in the Sco-Cen OB association. With respect to the mass function, some indications of a dependence of the results on the physical conditions of star formation is found in recent analyses (Luhman et al. 2003; Pozzo et al. 2003), whereas recent observations of PMS stars in the intermediate mass range in 30 Dor (LMC) suggest no important differences between star formation in clusters in the Milky Way and outside it (Brandner et al. 2001).

In this paper we present the results for ten southern clusters, mostly located in the Carina-Sagittarius spiral arm of the Milky Way. This research is the continuation of a project, centred on the issues of interest already mentioned in this section, which has produced results for some clusters, mainly located in the Cygnus region and surroundings (see Delgado et al. 2004, and references therein). The main point in this research consists in assigning membership to individual candidate PMS stars on the basis of theoretical isochrone fitting on various CMD, in the same way as classical multicolour photometry is used to estimate membership of main sequence (MS) stars by means of ZAMS fitting. The procedure has been shown to perform better, when the reddening of the parent cluster is lower (Delgado \& Alfaro 2000). The project aims at obtaining homogeneous sample of PMS stars at different locations in the Galactic disk. It should provide a sample for future spectroscopic studies of individual objects, as well as for analysing (i) key issues of star formation in clusters, such as age and relative spatial distributions of MS and PMS members and (ii) the presence and dissipation time scales of circumstellar disks. Other points of interest are the assessment of

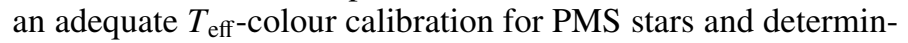
ing to what extent the application of calibrations valid for dwarfs is adequate for these stars (Delgado et al. 1998; Mamajek et al. 2002). Very interestingly, Lyo et al. (2004) provide an observational isochrone from $\eta$-Cham observations, although for PMS stars with spectral types later than K7.

Section 2 contains a description of the observations and of the reduction and calibration procedures. A comparison with the previously published photometry of some of the clusters is presented. In Sect. 3 we describe and discuss the methodology used for determining the main physical properties of the host clusters, as well as the membership assignments and ages for post-MS and PMS stars in the observed area. Matches with the Two Micron All Sky Survey (2MASS) ${ }^{1}$ catalogue and the resulting astrometry and near-infrared colour indices of the candidate members are addressed. Section 4 contains a brief summary of how the catalogue has been elaborated and a description of its contents.

\footnotetext{
1 http://www.ipac.caltech.edu/2mass/
} 
Table 1. Observed clusters.

\begin{tabular}{|c|c|c|c|c|c|c|c|}
\hline Cluster & & $\begin{array}{r}\text { RA } \\
\text { Epocl }\end{array}$ & h 2000 & Dec & $\begin{array}{c}l \\
\text { de }\end{array}$ & $b$ & Ref. $^{1}$ \\
\hline NGC 2367 & 720 & 004.54 & -2153 & 02.7 & 235.65 & -3.84 & $\bar{a}$ \\
\hline NGC 3293 & 1035 & 548.77 & -5813 & 28.1 & 285.85 & 0.07 & $\mathrm{~b}$ \\
\hline Collinder 228 & 1043 & 301.26 & -6000 & 44.8 & 287.52 & -1.03 & $\mathrm{c}$ \\
\hline Hogg 10 & 1110 & 55.02 & -6022 & 18.1 & 290.80 & 0.10 & $\mathrm{~d}$ \\
\hline Hogg 11 & 1111 & 32.63 & -6022 & 49.3 & 290.89 & 0.14 & $\mathrm{e}$ \\
\hline Trumpler 18 & 1111 & 26.29 & -6040 & 19.1 & 290.99 & -0.14 & $\mathrm{f}$ \\
\hline NGC 3590 & 1112 & 55.22 & -6046 & 30.7 & 291.21 & -0.18 & d \\
\hline NGC 4103 & 1206 & 42.65 & -6114 & 26.8 & 297.57 & 1.18 & $\mathrm{~g}$ \\
\hline NGC 4463 & 1229 & 57.47 & -6447 & 55.7 & 300.65 & -2.01 & $\mathrm{~h}$ \\
\hline NGC 5606 & 1427 & 47.30 & -5938 & 34.8 & 314.84 & 0.99 & $\mathrm{i}$ \\
\hline
\end{tabular}

1 a: Vogt \& Moffat (1972). b: Turner et al. (1980). c: Carraro \& Patat (2001). d: Moffat \& Vogt (1975). e: Clariá (1976). f: Vázquez \& Feinstein (1990). g: Wesselink (1969). h: Moffat \& Vogt (1973). i: Vázquez \& Feinstein (1991).

\section{Observations}

The observations were carried out with the YALO $^{2}$ telescope, operating at Cerro Tololo Observatory (CTIO), with the ANDICAM $^{3}$ camera, in two campaigns during December 2000 and May 2001. The optical channel of ANDICAM incorporates an array of $2024 \times 2024$ pixels, with a plate scale of $0.3^{\prime \prime} /$ pixel. The $B V R_{\mathrm{C}} I_{\mathrm{C}}$ filters were mounted, together with the Strömgren ultraviolet $u$ filter, instead of the commonly-used $U$ passband from the Johnson system.

Several frames were secured through each band for every cluster field (Table 1), with integration times for short exposures of $5 \mathrm{~s}$ for $B V R I$ bands and $10 \mathrm{~s}$ for the $u$ band, and long exposures of $1200,600,300,300$, and $700 \mathrm{~s}$, for the $u B V R I$ bands, respectively. Three fields from the catalogue of standard stars by Landolt (1992) were also observed. The fields of SA98-650, and Rubin 149, observed in the 2000 campaign, include 24 standard stars. The fields from the 2001 campaign, SA110-229, and PG1657+078, contain 8 standards. All these fields were observed every night at a minimum of three separated airmasses, in every band. As mentioned above, the ultraviolet filter at our disposal was the $u$ Strömgren filter. No measurements of the standard fields were secured through this filter. The calibration of the $(U-B)$ colour index is feasible if we dispose of accurate published Johnson $(U-B)$ values in the field, and a reliable zeropoint correction between instrumental and "standard" values can be determined.

The different frames were reduced with the routines in the $\mathrm{IRAF}^{4}$ package. Aperture corrections and atmospheric extinction corrections were applied to the magnitudes in each frame. Average extinction coefficients for CTIO $^{5}$ were used. Instrumental magnitudes were then calculated as weighted averages of the magnitudes in all frames. The weights used are the inverse of the squared PSF photometric errors.

${ }^{2}$ YALO is the Yale-AURA-Lisbon-Ohio consortium (Bailyn et al. 1999). http://www . astronomy . ohio-state. edu/YALO/

${ }^{3}$ http://www-astronomy.mps.ohio-state.edu/ depoy/ research/instrumentation/andicam/andicam.html

${ }^{4}$ The Image Reduction and Analysis Facility (IRAF) is distributed by the national Optical Astronomical Observatory, which is operated by the Association of Universities for Research in Astronomy, Inc. (AURA) under cooperative agreement with the National Science Foundation.

${ }^{5}$ http://www.ctio.noao.edu/facil/s4/sec4.html\#b

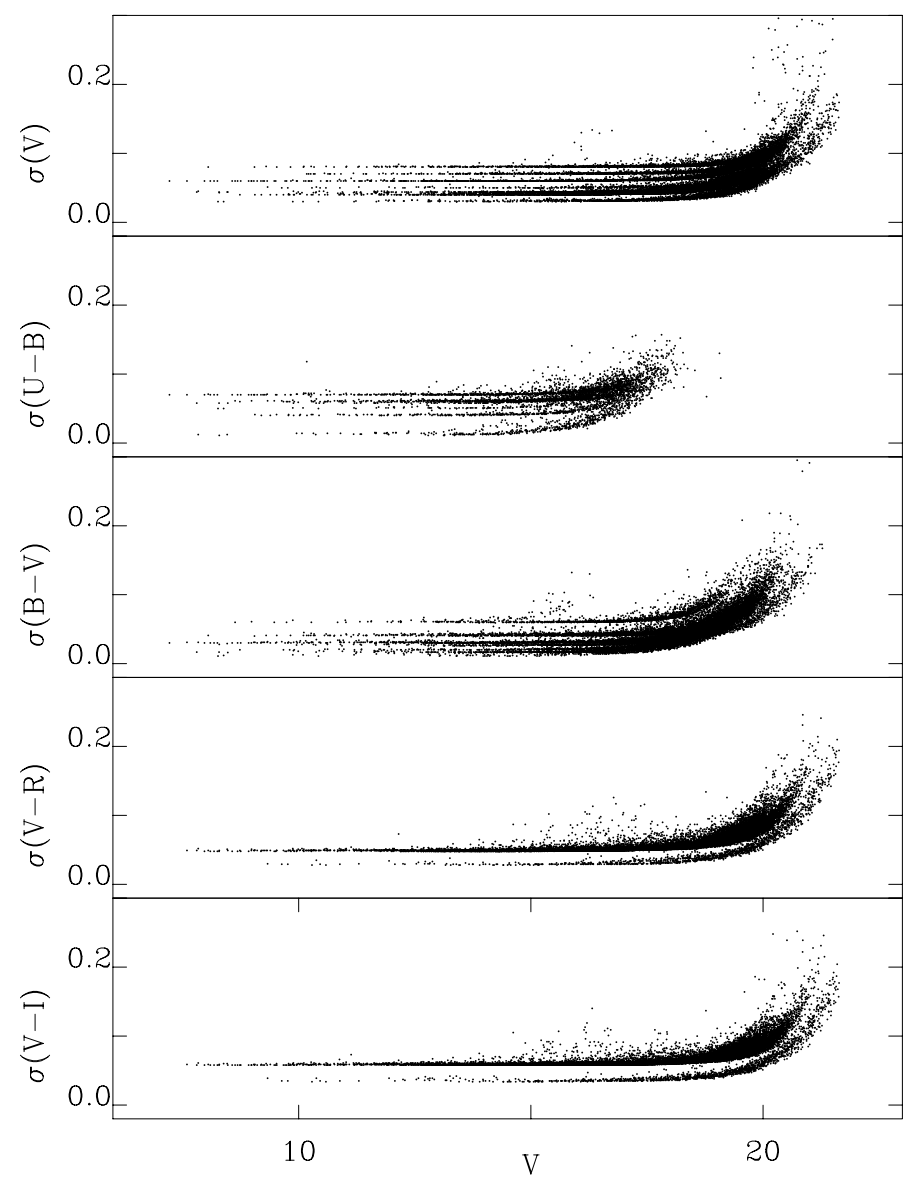

Fig. 1. Variation with $V$ in the total uncertainties of standard indices for all observed clusters.

The standard calibration of $U B V$ indices was carried out by direct correlation of instrumental values with published photometry for the individual clusters, included in the WEBDA ${ }^{6}$ database. These correlations produce accurate values for our purpose and offer a most reliable way of obtaining well-behaved $U B V$ indices (Delgado \& Alfaro 2000). The calibrations consist in determining zero-point corrections from the comparisons between instrumental and published values, with eventual colour-dependent terms after examining the residuals. The rootmean-squared deviation of the final residuals is adopted as the uncertainty on the calibration. The calibration of the $(V-R)_{\mathrm{C}}$ and $(V-I)_{\mathrm{C}}$ indices was performed with the help of the observed Landolt standard stars, using the classical linear model for the calibration equations (see Delgado et al. 1998). As before, the root-mean-squared deviations of the fits were taken as the uncertainty on the calibrations.

The total errors assumed for the final standard indices are quadratic sums of the photometric errors and calibration uncertainties as described above. In Table 1, we list the target clusters' equatorial and galactic coordinates and the photometric study of reference used to compute the $U B V$ calibration. In Fig. 1 we represent the variation in the uncertainties in a joint plot with $V$ for all the measured stars together. The different levels, appreciable at the brightest magnitudes, reproduce the different uncertainties of the calibration for different clusters.

A comparison between our absolute photometry and those published for the clusters with CCD data, provides the values listed in Table 2. In this table we list the average differences

\footnotetext{
${ }^{6}$ http://www . univie.ac. at/webda//webda.html
} 
Table 2. Comparison to published CCD photometry.

\begin{tabular}{lrrrrrr}
\hline \hline Cluster & $(V)$ & $(U-B)$ & $(B-V)$ & $(V-R)$ & $(V-I)$ & Ref. $^{1}$ \\
\hline NGC 3293 & 0.00 & -0.04 & -0.01 & -0.01 & -0.00 & $\mathrm{a}$ \\
& 19 & 11 & 11 & 21 & 10 & \\
NGC 4103 & 0.04 & -0.03 & -0.02 & 0.04 & -0.03 & $\mathrm{~b}$ \\
& 13 & 13 & 13 & 12 & 14 & \\
NGC 4103 & 0.04 & & 0.06 & & 0.04 & $\mathrm{c}$ \\
& 9 & & 12 & & 8 & \\
NGC 5606 & 0.02 & 0.01 & -0.02 & 0.06 & 0.05 & $\mathrm{~d}$ \\
& 12 & 11 & 16 & 10 & 9 & \\
Collinder 228 & -0.02 & -0.01 & 0.00 & 0.03 & 0.04 & $\mathrm{e}$ \\
& 11 & 8 & 9 & 8 & 11 & \\
\hline
\end{tabular}

${ }^{1}$ a: Baume et al. (2003). b: Sagar \& Cannon (1997). c: Sanner et al. (2001). d: Vázquez et al. (1994). e: Carraro \& Patat (2001).

in the first row, and the root-mean-squared (rms) deviations for each cluster in hundreths of magnitude in the second row. The high dispersion in some cases originates in the large errors for faint stars. We do, however, note the absence of significant systematic differences between our adopted colours and the published ones. Restricting the comparison to stars brighter than $V=20$, the rms deviation of the differences decreases below 0.1 in all cases.

For Collinder 228, the $U B V$ calibration in terms of published photoelectric values (Feinstein et al. 1976; Turner \& Moffat 1980) provides unreliable results. Just for this cluster, the CCD values by Carraro \& Patat (2001) were then used to calibrate the $V,(U-B)$, and $(B-V)$ indices.

\section{Construction of the catalogue}

Our main objective is to detect "bona fide" PMS cluster-member candidates from multicolour photometry in the area of the cluster. Candidates not only need to be assigned as cluster members, but also have to be located in a region of the CM diagrams, which are compatible with a PMS evolutionary stage. We therefore devote some room to explaining the methodology used to assign cluster membership to the individual stars and to distinguishing between post-MS and PMS members. This information can be obtained from the comparative analysis of the observational CC and $\mathrm{CM}$ diagrams, and the comparison between observational and model diagrams.

\subsection{Colour excess and distance: MS membership}

The first step in the procedure of membership assignment is the calculation of colour excess and distance for MS stars. These values are to be used as reference to estimate the membership for PMS candidate-members. We note that most of the clusters in our sample do not have membership assignments for stars in the the region of the CMD where PMS members would be expected. Only NGC 3292, the most-studied object, has membership assignments for stars fainter than $M_{\mathrm{V}}=2$ (Baume et al. 2003). Some of our observed stars in Collinder 228 could be included in the $B V R I H \alpha$ photometric study of a stellar field in the Carina region by Hägele et al. (2003). However, this study does not provide published or accessible data that could be compared to our results.

To calculate colour excess and absolute magnitude from the $(U-B),(B-V) \mathrm{CC}$ diagram, we used the standard values of the reddening parameters, $\alpha \equiv E(U-B) / E(B-V)=0.72$, $R \equiv A_{V} / E(B-V)=3.1$. For NGC 3293, the detailed study of
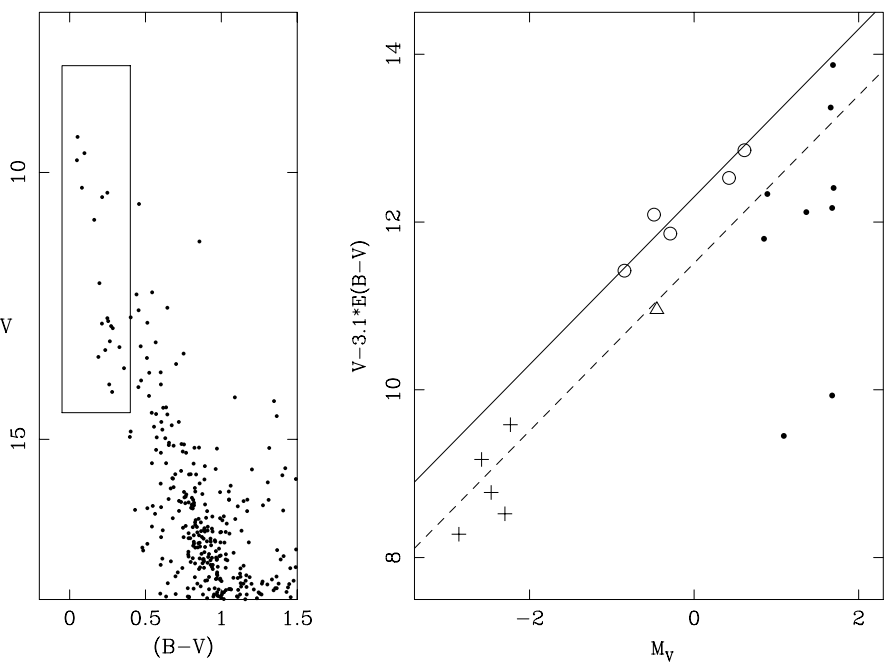

Fig. 2. Example of selection of probable unevolved MS members. The left panel shows the tentative selection of upper MS members in the $V,(B-V)$ diagram of NGC 2367 (see text). In the right panel, the quantity $V-3.1 \times E(B-V)$ is plotted for these stars versus $M_{\mathrm{V}}$. The lines indicate published values of the distance modulus: Vogt \& Moffat (1972) (continuous line) and Kharchenko (2005) (broken line). Circles are stars selected from the plot as probable, unevolved MS members. Triangles and crosses are stars selected as post-MS members. Crosses mark the stars used to estimate the post-MS age of the cluster (see text).

the reddening slope in the cluster field by Turner et al. (1980), suggests the value $\alpha=0.76$, so it has been adopted here.

\subsubsection{Selection of probable, unevolved MS members}

Our procedure for first selecting the upper MS probable members is to use the location of stars in the CM and CC diagrams, as described in Delgado et al. (1998). We start with the assumption that all clusters have a certain number of unevolved MS members with spectral types bluer than A0. A tentative selection of these stars is made visually in the $V,(B-V) \mathrm{CM}$ diagram, also checking that the stars are congruently located in the other CM diagrams. This procedure is shown in Fig. 2, where the example of NGC 2367 is plotted. For the stars tentatively selected (left panel) a colour excess and a corresponding absolute magnitude are calculated by means of the calibrated ZAMS line (SchmidtKaler 1982).

The quantity $V-3.1 \times E(B-V)$ is then plotted versus $M_{\mathrm{V}}$, also considering previously published values of cluster parameters. In the right panel of Fig. 2, we see this plot for the tentative member stars, with the two different published values for the distance modulus of the cluster indicated by two different lines (Vogt \& Moffat 1972; Kharchenko 2005, also adopted in WEBDA). In view of this plot, we select stars as probable unevolved MS members and others as evolved post-MS members, which will be used later to perform a quantitative estimate of the cluster age. The selection performed in these plots is based on how stars that evolved away from the ZAMS will show a lower distance modulus than the unevolved ones. In the same way, rotating or binary stars would have the same effect upon the DM calculation so are also rejected, as for the star marked with a triangle in the right panel of Fig. 2.

For most clusters in our sample, this procedure provides a reliable selection of upper non evolved MS members and post-MS members. For some of them, the selection of a cluster sequence is not unambiguous. In Fig. 2 we show the case of Trumpler 18 as 

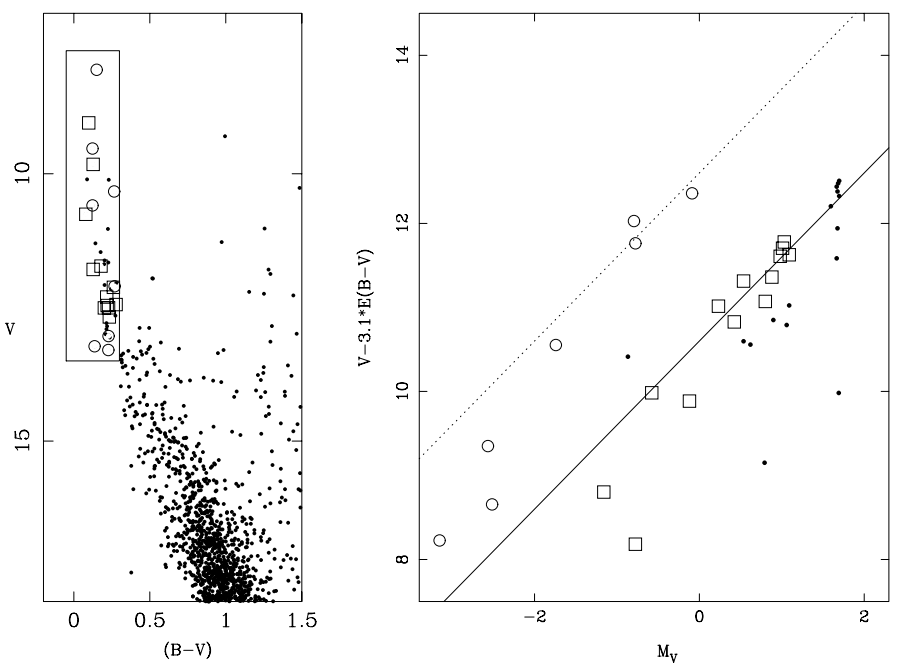

Fig. 3. Same plots as in Fig. 2, for Trumpler 18. The stars in the two possible sequences are plotted with different symbols. The selection of upper probable MS cluster members is denoted with squares. The lines represent distance moduli of 10.6 (continuous) and 12.7 (dotted).

an example, where two associations seem to overlap in the same field, at different distances. The selected possibility, according to previously published values, is shown with the larger distance modulus that would account for a star sequence located further away.

The selection of probable unevolved MS members is obviously of central importance for the membership analysis. The solutions adopted here, based on the plots like the one in Figs. 2 and 3 are aimed at obtaining a basis for further PMS membership analysis, and they follow the appearance of our own CM diagrams, together with previously published results. On the other hand, there are interesting features in the CM diagrams of several clusters, suggesting the presence of several groupings at different distances in the same line of sight. This fact is apparent for lines of sight crossing the Carina spiral arm at different positions. These features can be guessed, for example, in the plots shown in both Figs. 2 and 3. In this paper we only elaborate a catalogue of candidate PMS cluster members. The discussion of these features in the CM diagrams of the target clusters is postponed for a future paper.

The method is applied to every cluster, and provides average values of colour excess and distance modulus for the probable unevolved MS members. These are listed in Table 3 with their rms deviations, together with the results derived by other authors. The values in Table 3 agree in most cases with the published results. There are, however, some appreciable differences, as in the case of Hogg 10, where the three published values of colour excess are noticeably higher than our value. This difference is due to our restricting the selection of probable unevolved MS members to avoid including binaries or evolved stars as much as possible. But we also generally obtain a more complete coverage of the cluster MS, whereas the photoelectric studies used contain only a few stars. In the particular case of Hogg 10, for example, these are the ones with highest reddening.

\subsubsection{Membership assignment for possible MS members}

The average values of colour excess and distance modulus for the probable unevolved MS members are used to estimate membership for the remaining stars.
Values of colour excess and distance modulus are calculated for each star by comparing their indices with the ZAMS reference line (Schmidt-Kaler 1982) in the $(U-B),(B-V) \mathrm{CC}$ diagram. The representative point of each star is shifted in the $\mathrm{CC}$ diagram, with the adopted reddening slope, and surrounded by an ellipse of semiaxes given by the uncertainties in $(U-B)$ and $(B-V)$ (Fig. 1). The minimum distance at every intersection of this ellipse with the reference line provides a colour excess $E(B-V)$ and a particular value of the absolute visual magnitude $M_{\mathrm{V}}$, wich are used to calculate a value for the intrinsic distance modulus.

The calculation is visualised in Fig. 4, using three stars in the cluster NGC 3590 as an example. Figure 4 shows how more than one solution is possible for each star, depending on its location in the CC diagram. A star is then considered as a member if any one of the pairs of values $E(B-V), D M$ coincides, within the errors, with the average values from the probable unevolved MS members. In this comparison, the errors used are squared quadratic sums of the uncertainties plotted in Fig. 1, and the rms deviation of the average values (Table 3). In practice, when selecting MS members, we require that the colour excess be larger than the average value of the probable unevolved MS members minus the error and smaller than this value plus twice the error. When selecting PMS members, we simply require the colour excess be above the value of the probable unevolved MS members minus the error. We try in this way to favour the detection of possible members, PMS members in particular, which are more affected by reddening than the probable unevolved MS members.

We note that a star may already have one calculated value for the colour excess that fulfils the requirements for membership, prior to the distance modulus comparison. In considerations below we refer to these stars as reddening candidates. In fact, this constitutes the first membership filter. Obviously, all stars assigned as members are first reddening candidates, but only stars that fulfil this condition and the agreement of the corresponding distance modulus are kept as candidate members.

\subsubsection{Age estimate for post-MS stars}

The age of the cluster is estimated from comparing of the CM diagrams with theoretical post-MS isochrones. A simple quantitative procedure is used, which provides a systematic age estimate instead of the commonly-used guess from a visual comparison between data and isochrones. A similar procedure has been used by Trullols \& Jordi (1997).

As done for the probable unevolved MS members, the postMS probable members used in the calculations are selected from plots like those shown in Figs. 1 and 2, also considering the consistent location of every star in all CM diagrams, and helped by results from previous studies. As is known, in addition to observational errors and to metallicity variations, which are not expected in the studied young clusters, the presence of rotation and binarity cause a broadening of the cluster's main sequence (see for instance, Fernandes et al. 1996). This would lead to overestimating the cluster age. In our selection we try to avoid stars that could be affected by these effects.

In the calculation we use isochrones from the Padova group (Girardi et al. 2002), for LogAge(yr) values of 6.6, 6.7, 6.8, 6.9, 7.0, 7.2, 7.4, and 8. The isochrones are first shifted in all CM diagrams to account for the colour excess and distance moduli calculated for the probable unevolved MS members. Furthermore, the colour calibration used to translate the theoretical tracks to the observational plane present offsets with respect to the ZAMS of Schmidt-Kaler (1982) or Kenyon \& Hartmann (1995), which 
Table 3. Comparison with published results.

\begin{tabular}{|c|c|c|c|c|}
\hline Cluster & $E(B-V)$ & $D M$ & Log(Age) & Reference \\
\hline \multirow[t]{4}{*}{ NGC 2367} & 0.33 & 11.51 & 6.74 & WEBDA \\
\hline & 0.35 & 12.28 & & Vogt \& Moffat (1972) \\
\hline & 0.33 & 11.51 & 7.01 & Kharchenko et al. (2005) \\
\hline & $0.38 \pm 0.06$ & $12.3 \pm 0.2$ & $6.6 \pm 0.3$ & This work \\
\hline \multirow[t]{8}{*}{ NGC 3293} & 0.26 & 11.83 & 7.01 & WEBDA \\
\hline & 0.29 & 11.99 & 6.70 & Turner et al. (1980) \\
\hline & 0.31 & 12.10 & 7.00 & Feinstein \& Marraco (1980) \\
\hline & & 11.95 & & Shobbrook (1983) \\
\hline & 0.25 & $11.8-12.6$ & & cited by Shobbrook (1983) \\
\hline & 0.22 & 12.20 & 6.90 & Baume et al. (2003) \\
\hline & 0.25 & 11.96 & 6.94 & Kharchenko et al. (2005) \\
\hline & $0.29 \pm 0.04$ & $12.0 \pm 0.2$ & $6.8 \pm 0.4$ & This work \\
\hline \multirow[t]{7}{*}{ Collinder 228} & 0.34 & 11.71 & 6.83 & WEBDA \\
\hline & 0.39 & 12.00 & 6.70 & Feinstein et al. (1976) \\
\hline & 0.32 & 12.17 & & Turner \& Moffat (1980) \\
\hline & 0.30 & 11.39 & 6.90 & Carraro \& Patat (2001) \\
\hline & 0.37 & 12.45 & & Massey et al. (2001) \\
\hline & 0.26 & 12.21 & 6.68 & Kharchenko et al. (2005) \\
\hline & $0.32 \pm 0.12$ & $12.0 \pm 0.3$ & $6.7 \pm 0.2$ & This work \\
\hline \multirow[t]{4}{*}{ Hogg 10} & 0.46 & 11.25 & 6.78 & WEBDA \\
\hline & 0.46 & 11.71 & & Moffat \& Vogt (1975) \\
\hline & 0.49 & 12.24 & 7.55 & Claria (1976) \\
\hline & $0.36 \pm 0.05$ & $11.9 \pm 0.2$ & $6.8 \pm 0.5$ & This work \\
\hline \multirow[t]{4}{*}{ Hogg 11} & 0.32 & 11.78 & 7.08 & WEBDA \\
\hline & 0.32 & 11.81 & & Moffat \& Vogt (1975) \\
\hline & 0.24 & & 6.90 & Ahumada et al. (2001) \\
\hline & $0.30 \pm 0.05$ & $11.9 \pm 0.2$ & $6.7 \pm 0.3$ & This work \\
\hline \multirow{5}{*}{ Trumpler 18} & 0.32 & 10.66 & 7.19 & WEBDA \\
\hline & 0.29 & 10.54 & & Moffat \& Vogt (1975) \\
\hline & $0.29-0.47$ & $10.54-11.84$ & & Cited by Claria (1976) \\
\hline & 0.31 & 10.66 & 7.77 & Kharchenko et al. (2005) \\
\hline & $0.30 \pm 0.04$ & $10.6 \pm 0.2$ & $7.5 \pm 0.5$ & This work \\
\hline \multirow[t]{5}{*}{ NGC 3590} & 0.45 & 11.09 & 7.23 & WEBDA \\
\hline & 0.51 & 11.57 & & Moffat \& Vogt (1975) \\
\hline & 0.51 & 11.79 & 7.56 & Claria (1976) \\
\hline & 0.45 & 11.09 & 7.55 & Kharchenko et al. (2005) \\
\hline & $0.52 \pm 0.08$ & $11.7 \pm 0.2$ & $7.2 \pm 0.2$ & This work \\
\hline \multirow[t]{5}{*}{ NGC 4103} & 0.29 & 11.06 & 7.39 & WEBDA \\
\hline & 0.31 & 11.51 & 7.48 & Sagar \& Cannon (1997) \\
\hline & 0.26 & 11.70 & 7.30 & Sanner et al. (2001) \\
\hline & 0.28 & 11.11 & 7.59 & Kharchenko et al. (2005) \\
\hline & $0.32 \pm 0.04$ & $11.5 \pm 0.2$ & $7.3 \pm 0.2$ & This work \\
\hline \multirow[t]{4}{*}{ NGC 4463} & 0.43 & 10.11 & 7.51 & WEBDA \\
\hline & 0.44 & 11.77 & & Moffat \& Vogt (1973) \\
\hline & 0.52 & 10.11 & 7.97 & Kharchenko et al. (2005) \\
\hline & $0.39 \pm 0.04$ & $11.1 \pm 0.1$ & $7.3 \pm 0.5$ & This work \\
\hline \multirow[t]{6}{*}{ NGC 5606} & 0.47 & 11.28 & 7.08 & WEBDA \\
\hline & 0.49 & 11.40 & & Moffat \& Vogt (1973) \\
\hline & 0.51 & 11.90 & 6.82 & Vazquez et al. (1994) \\
\hline & 0.31 & & 6.65 & Ahumada et al. (2001) \\
\hline & 0.47 & 11.28 & 6.84 & Kharchenko et al. (2005) \\
\hline & $0.49 \pm 0.05$ & $11.8 \pm 0.2$ & $7.0 \pm 0.3$ & This work \\
\hline
\end{tabular}

coincide quite closely. When using the isochrones for age estimates, these offsets are previously corrected.

For each involved star, the difference in colour with respect to every isochrone is measured in the four CM diagrams available. These differences are then either interpolated or extrapolated to the age value that would fit the star position exactly. This provides a nominal age value for each star, and all these values are then averaged to give the post-MS age estimate for the cluster. The average values and their rms deviations are listed in
Table 3, together with other clusters parameters, and compared to published values.

\subsection{PMS membership}

We distinguish between stars for which a colour excess can be calculated in the CC diagram and stars that do not have this possibility, either because of lacking $U$ measurement, the most 


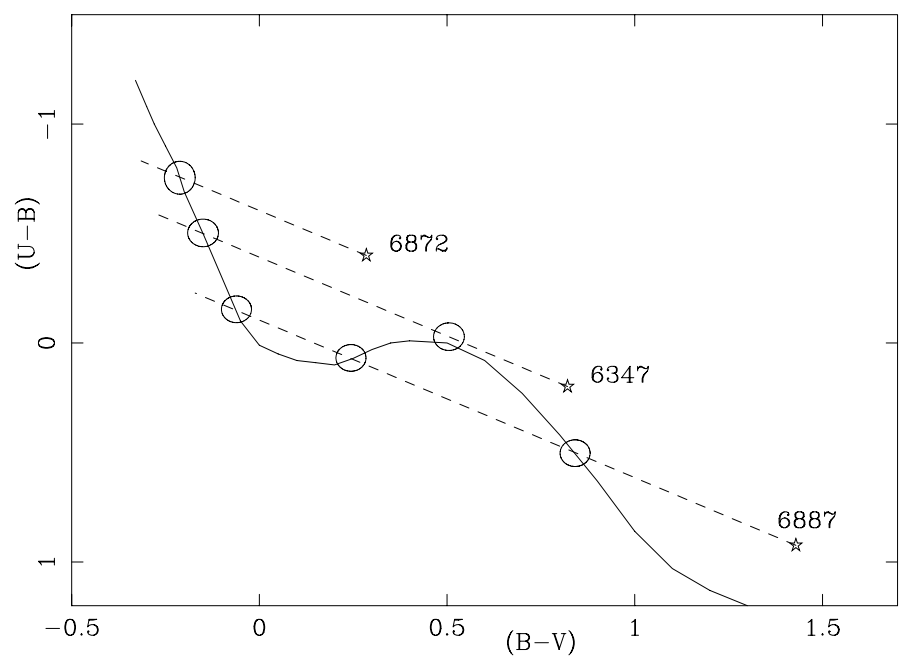

Fig. 4. Example of the colour excess calculation in the $(U-B),(B-$ $V)$ diagram. Three stars in NGC 3590 with different types of solutions are plotted, together with the ZAMS (continuous line). Broken lines represent the shifts with the slope $E(U-B) / E(B-V)=0.72$. Ellipses represent the photometric uncertainties of the respective points, plotted in Fig. 1.

common case, or because the shifting in the CC diagram does not provide any valid intersection with the reference lines.

For stars with $(U-B)$ measurement, the calculation proceeds as explained above. These stars were also compared with the PMS isochrones, taken from three different models (D'Antona \& Mazzitelli 1997; Palla 1999; Siess et al. 2000, respectively referred to in the following as D97, P99, and S00). The $(U-B)$ vs. $(B-V)$ relation for these isochrones was the same as the one used for the ZAMS (Kenyon \& Hartmann 1995). The colour excesses were therefore the same, whereas the distance moduli were different, depending on the model and on the age of the isochrone. We used model isochrones of ages from 1 to $10 \mathrm{Myr}$ in steps of 1 Myr for the S00 models and 2 Myr for D97 and P99 models. Therefore, we obtained up to 20 possible DM values, from 10 S00 isochrones, 5 P99 isochrones, and 5 D97 isochrones. A star was then assigned as a PMS member if, in addition to the colour excess requirement for PMS candidates (Sect. 3.1.2), one or more of these DM values agreed within the errors with the average value of the probable MS unevolved members. Obviously, this membership assignment wss done simultaneously in the two CM diagrams $V,(U-B)$ and $V,(B-V)$.

For stars without a valid $(U-B)$ value, the $C C$ diagram cannot be used, and we do not have calculated values of colour excess and distance modulus to compare with reference lines. For these stars, only PMS membership was investigated. To assign PMS membership to these stars, we assumed every star was affected by the colour excess calculated for the probable unevolved MS members. To compare stars with isochrones in the $V,(V-R)$ and $V,(V-I) \mathrm{CM}$ diagrams, we used reddening slopes for adequate achieving of a good ZAMS fitting of the probable unevolved MS members in the respective diagrams. These values for the reddening slopes are in good agreement with the values from a standard reddening law (Cardelli et al. 1999). The PMS isochrones are shifted with these values in the CM diagrams, and the comparison with the data gives a distance modulus for every star relative to each isochrone. A star has a membership assignment (with respect to an isochrone) if the obtained distance modulus coincides, within the errors, with the average value for the probable MS unevolved members. In this procedure, a star

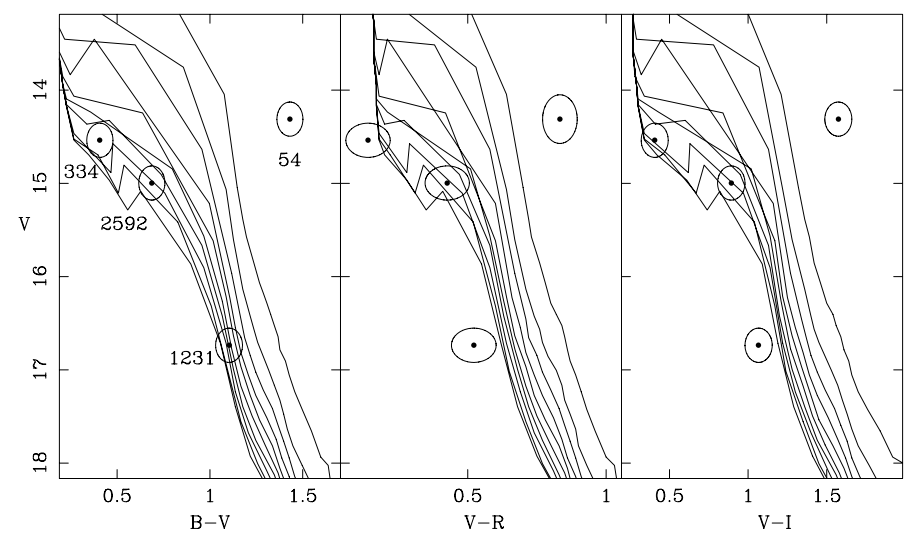

Fig. 5. Example of PMS members selection in the CM diagrams of NGC 3293. Star \# 54, non member; \#1231, member in the $V,(B-V)$ diagram; \#334, member in three CM diagrams, with different ages; \#2592, member in all three diagrams, with age differences among them below $1 \mathrm{Myr}$. The axes of the ellipses around each point reproduce the squared quadratic sums of final uncertainties (Fig. 1) and the rms deviation of cluster parameters for the probable unevolved MS members (Table 3). Isochrones of ages 1 to $10 \mathrm{Myr}$ from Siess et al. (2000) are plotted.

can have a membership assignment in one, two, or all three CM diagrams involved.

To clarify this procedure of member selection, we show examples in the CM diagrams of NGC3293 in Fig. 5 of stars with different qualities of membership assignment. The ellipses around each point reproduce the errors explained above, used for comparison: squared quadratic sums of the uncertainties plotted in Fig. 1, and rms deviation of the average colour excess and distance modulus for the probable unevolved MS members. The isochrones plotted in this example are from Siess et al. (2000).

In both methods of membership assignment, the errors used in the comparisons are the squared quadratic sums of the uncertainties plotted in Fig. 1, and the rms deviation of the average values for the probable unevolved MS members. As can be appreciated in the example of Fig. 5, the errors are usually larger than the separation between two consecutive isochrones. As a result, every star can be a candidate member with respect to several PMS isochrones. The average value of the corresponding ages is taken as the age of the PMS candidate. The average value of the ages for all assigned members and its rms value are adopted as the age and uncertainty of the PMS sequence of the cluster, as obtained from the particular CM diagram and model being compared.

Two more points have to be covered before finally selecting the candidate PMS members. First, the procedure described above for analysing the membership of stars without a valid $(U-B)$ index was also applied to stars with a valid $(U-B)$ value, and qualified as reddening candidates (see the last paragraph in Sect. 3.1.2). For stars without a $(U-B)$ index, the average colour excess of the probable unevolved MS members was assumed, as explained above. For reddening candidates, their own calculated colour excess value was used instead. In this way, PMS membership assignment can be established for a star with respect to several combinations of three CM diagrams, and even with respect to four CM diagrams. This is indeed the case of a star with an eventual assignment based on its calculated colour excess and distance modulus (as mentioned above, this obviously means simultaneous assignment in the $V,(U-B)$ and $V,(B-V) \mathrm{CM}$ diagrams), and also assigned as a member from its location in the $V,(V-R)$ and $V,(V-I) \mathrm{CM}$ diagrams. 


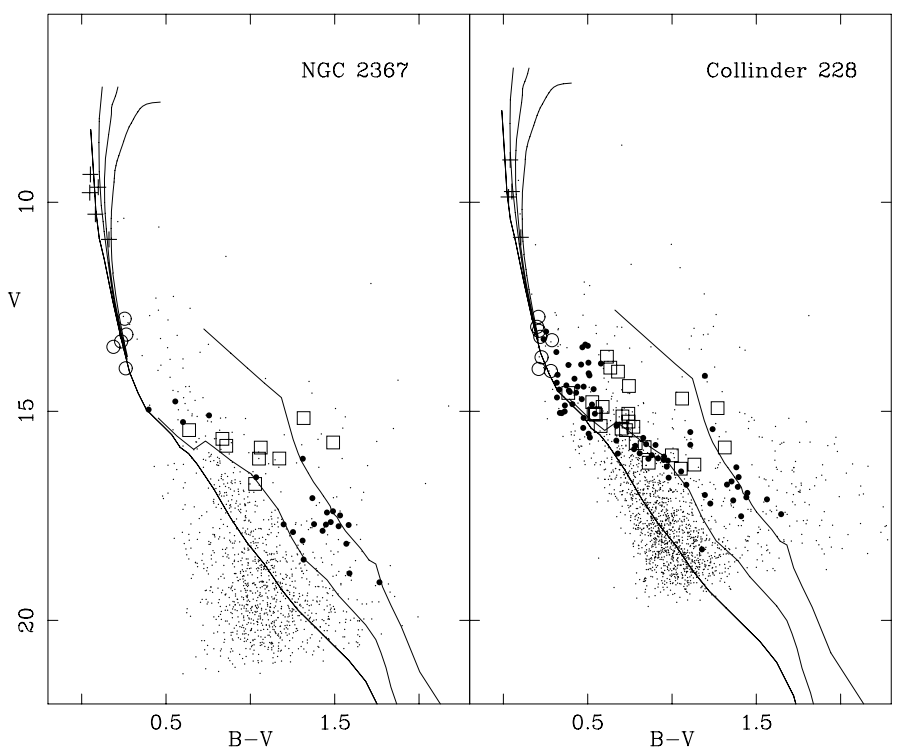

Fig. 6. $V,(B-V) \mathrm{CM}$ diagrams of NGC 2367 and Collinder 228, two of the youngest clusters in our sample. Circles represent the probable unevolved MS members. Crosses are the post-MS probable members selected for the quantitative age estimate. Large dots represent PMS members in respect to the S00 model, and selected from three CM diagrams. Squares are PMS members assigned in all four CM diagrams. Small dots are the remaining observed stars. The ZAMS line, post-MS isochrones by G02, for LogAge(yr) 6.6, 7.0, and 7.4, and S00 PMS isochrones for LogAge(yr) 6 and 7, are plotted for reference.

Second, the procedure for membership assignment may lead, for some stars with a valid $(U-B)$ value to an assignment both as MS and PMS members. This will happen for stars located in the region of the CM diagrams where the ZAMS and the oldest PMS isochrones merge. In these cases, we adopted the PMS assignment for stars with spectral types later than $\mathrm{A} 0\left(M_{\mathrm{V}}=1.3\right.$ on the ZAMS) and the MS assignment for stars earlier than this.

The final selection of candidate PMS members included only stars whith a membership assignment in at least three CM diagrams. Furthermore, to achieve a conservative estimate, we considered a further level of membership quality, with two more restrictions. A star is definitely considered as a possible PMS member if, in addition to being assigned in three CM diagrams, (i) its photometric errors (PSF errors) are below 0.05 in all five $U B V R I$ bands and (ii) the ages obtained from all three (or eventually four) $\mathrm{CM}$ diagrams coincide within a range of $1 \mathrm{Myr}$. The resulting assigned members are used to calculate the PMS age, as explained, and are the stars listed in our final catalogue.

The results of the selection procedure are shown in the $\mathrm{CM}$ diagrams of the clusters. Figures 6 to 10 show the $V,(B-V)$ $\mathrm{CM}$ diagrams for all clusters, compared to both post-MS (G02) and PMS (S00) isochrones. As mentioned before, three PMS model isochrones are used to calculate this membership assignment (D97, P99, and S00). Three different assignments and ages are therefore obtained for the PMS candidates in each cluster. In Figs. 6 to 10, we only plot the candidate PMS members with respect to S00 isochrones. In our final catalogue, we include assigned members with respect to any of the three model isochrones. In this respect, we have to mention that D97 isochrones cover only the faintest part of the CM diagrams, and they do not provide PMS membership assignment for stars earlier than G0.

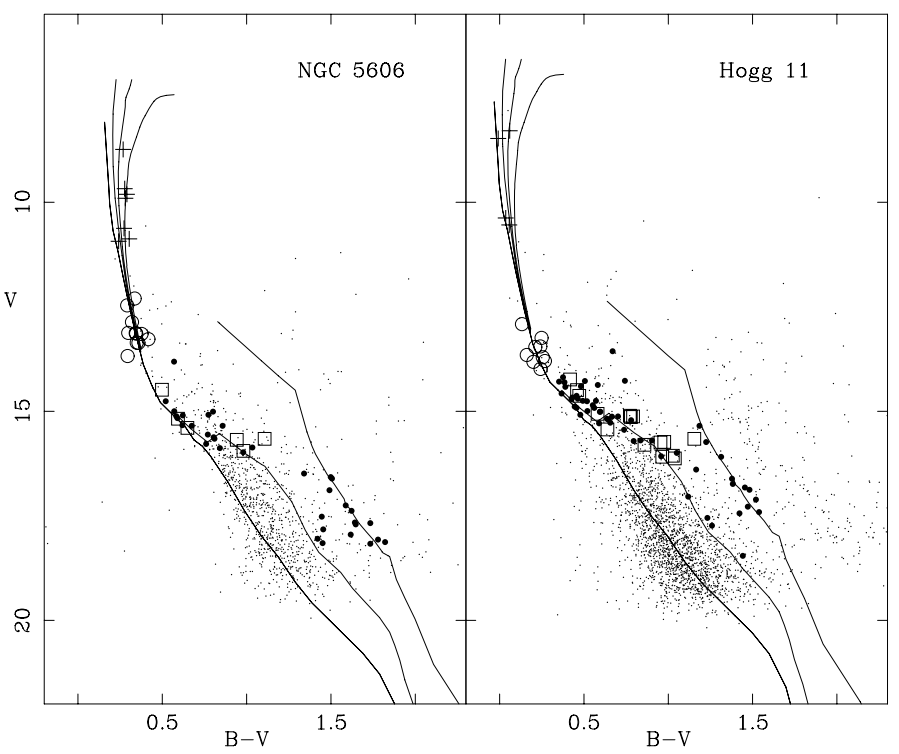

Fig. 7. The same as Fig. 3, for the clusters NGC 5606 and Hogg 11.

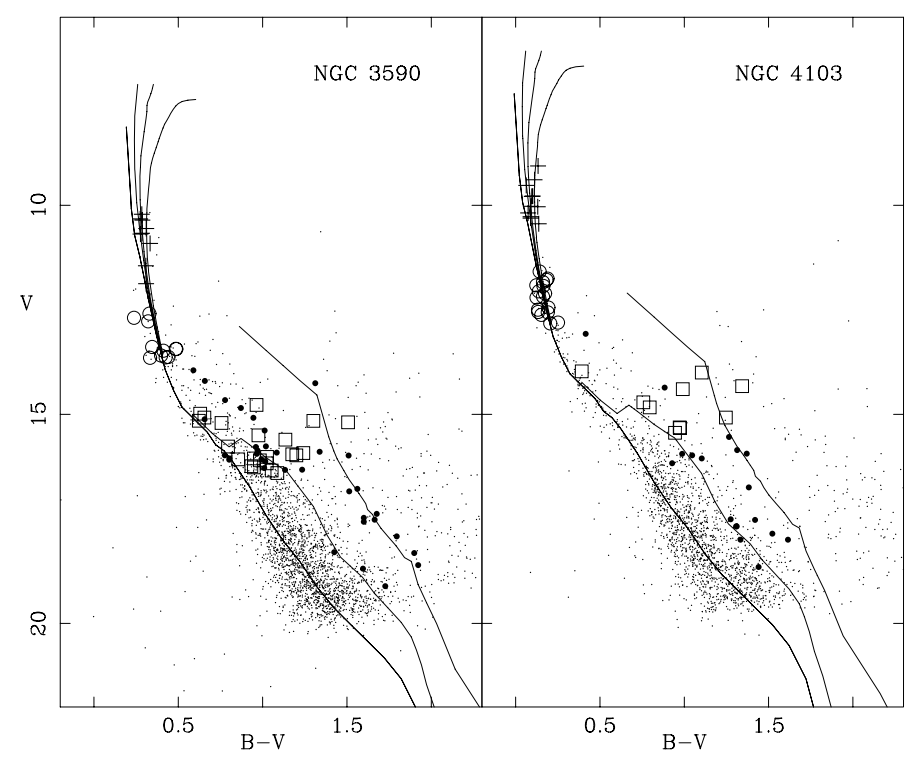

Fig. 8. The same as Fig. 3, for the clusters NGC 3590 and NGC 4103.

\subsection{Astrometry and $2 M A S S$ infrared data}

The pixel coordinates of our catalogue were transformed to equatorial coordinates with the IRAF tasks ccmap and xy2rd, using matching files with stars in common between our fields and corresponding fields in the 2MASS data base. The matching provides equatorial right ascension and declination for all the stars observed, and $J H K$ photometry for 15850 stars, while $68 \%$ of them have photometric quality flag A to $\mathrm{D}$ in all three $J H K$ bands (see the catalogue description at http://www.ipac.caltech.edu/2mass/releases/ allsky/doc/).

The values from the 2 MASS catalogue were transformed to the system of Bessel \& Brett (1988) with relations given by Carpenter (2001). These values are also included in the final catalogue. 


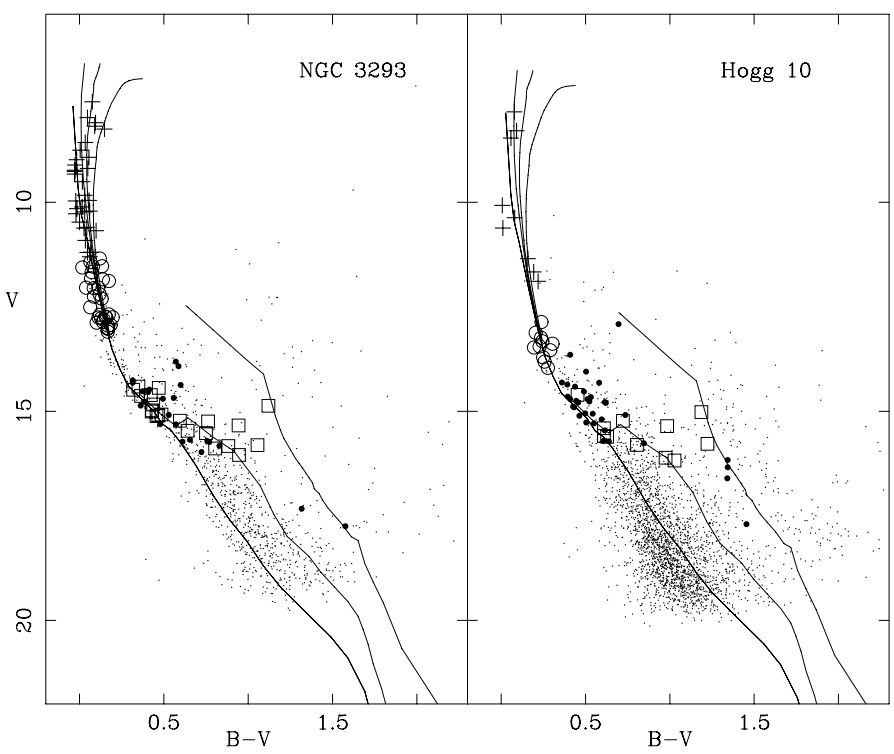

Fig. 9. The same as Fig. 3, for the clusters NGC 3293 and Hogg 10.

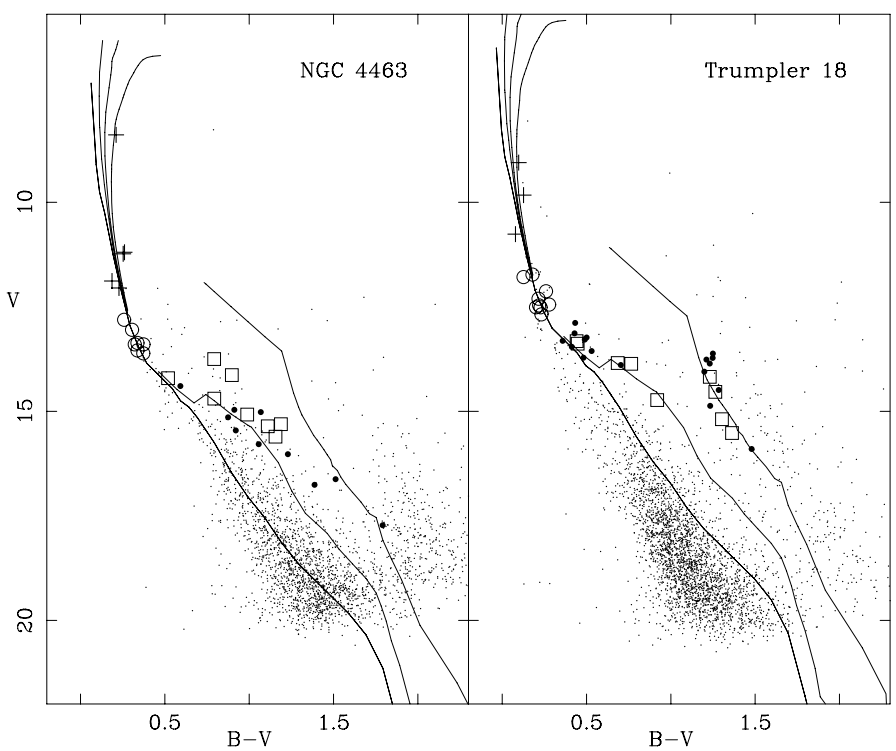

Fig. 10. The same as Fig. 3, for the clusters NGC 4463 and Trumpler 18.

\subsection{Additional remarks}

Cluster membership assignments derived from kinematic studies have always been considered more reliable than those obtained from photometric analysis. However, the determination of probable cluster members from analysing their location in the photometric diagrams has an ample and successful history, ever since its origin around the middle of the past century. We have profited from this experience, and with addition of new PMS evolutionary models, developed a methodology based on analysis of colour-colour and colour-magnitude diagrams to obtain a reliable sample of PMS cluster candidate members. This method was designed and developed in previous works and applied to several clusters (Delgado et al. 1998, 2000, 2004, 2006). Here, we use it to study a cluster sample, with homogeneous $U B V R_{\mathrm{C}} I_{\mathrm{C}}$ photometry, obtained at the same telescope and with the same instrumentation for all of them. The clusters are located mainly in the Carina complex of star formation (Alfaro et al. 1991), and they could therefore contain valuable information on the primary evolutionary stage of stars, inside clusters that form part of a larger region of coherent star formation in our Galaxy. The study of clusters in a wide age range, from 4 to $30 \mathrm{Myr}$ is of interest for analysing the possible sequential star formation and the resulting age spread inside the complex. Discussion of the age properties and of the members' spatial distribution and NIR properties is postponed to our next paper.

Some different sources of uncertainty that, among others, might be affecting our membership results are:

1. The $(U-B),(B-V)$ relation for PMS stars, may differ from the one for MS stars, and the reddening law for PMS stars, where unquantified amounts of remaining circumstellar dust and gas may be present, can also be different from the reddening relations describing extinction for MS stars (Delgado et al. 1998).

2. The values of the reddening slopes may indeed change between clusters (Sagar \& Cannon 1997; Guetter \& Turner 1997).

3. The eventual presence of high reddening makes it harder to distinguish PMS members and non-member stars (Chini \& Wargau 1990).

4. The uncertainties in the theoretical isochrones, which arise both from the model computation and from their translation to the observational plane as well.

It is not possible to evaluate the total uncertainty in the final classification due to these factors. We do, however, have arguments to support its reliability. First, we recall the restrictive conditions for the stars to be selected as members, which require not only a simultaneously consistent location in all CM diagrams, but also a good photometric accuracy in all five $U B V R I$ bands.

On the other hand, we have performed some follow-up spectroscopic studies of previously observed clusters, which provide results consistent with the photometric classification (Delgado et al. 1998, 1999, 2004). In the case of NGC 2362 (Delgado et al. 2006), a satisfactory agreement is reached between the described membership assignment and expectations from independently observed properties of the stars, such as $\mathrm{H} \alpha$ emission and $\mathrm{X}$ ray activity. We think that the effects of all the above-mentioned sources of uncertainty is decreased in the presence of not very high reddening, as is decidedly the case for NGC 2362, and also - although not so favourable - as for the clusters in the present sample.

Finally, we recall the comparison of our membership assignments with those by Baume et al. (2003) for NGC 3293, which shows a high degree of agreement: $89 \%$ of their 292 proposed members, also included in our observations, have some assignment by our procedure (either MS member or PMS member in at least one CMD).

\section{Catalogue and conclusions}

Multicolour $U B V R_{\mathrm{C}} I_{\mathrm{C}}$ photometry has been obtained for 26962 stars in the fields of 10 young clusters, 9 of them located in the Carina star formation complex of the Milky Way. Ages of the clusters, as estimated from their MS and post-MS probable members, range between Log Age(yr) 6.6 and 7.5. Matching of the pixel coordinates with the 2MASS catalogue provides absolute coordinates for DAY-I catalogue and $J H K$ photometry for the stars in common.

Analysis of the joint evidence from the $\mathrm{CC}$ and $\mathrm{CM}$ diagrams, together with the comparison of the observational $\mathrm{CM}$ diagrams to theoretical PMS and post-MS isochrones, led to estimates of both MS and PMS membership for individual stars 
Table 4. Catalogue of PMS candidates.

\begin{tabular}{|c|c|c|c|c|c|c|c|c|c|c|c|c|c|c|c|}
\hline \multirow[t]{2}{*}{$O C L-S \operatorname{tar} N o}$. & \multirow{2}{*}{$\begin{array}{c}\text { RA } \\
2000\end{array}$} & \multirow{2}{*}{$\begin{array}{c}\text { Dec } \\
2000\end{array}$} & \multirow[t]{2}{*}{$V$} & \multirow[t]{2}{*}{$U-B$} & \multirow[t]{2}{*}{$B-V$} & \multirow[t]{2}{*}{$V-R$} & \multirow[t]{2}{*}{$V-I$} & \multirow[t]{2}{*}{$K$} & \multirow[t]{2}{*}{$J-H$} & \multirow[t]{2}{*}{$H-K$} & \multirow[t]{2}{*}{ Mem } & \multirow[t]{2}{*}{$A_{V}$} & \multicolumn{3}{|c|}{ Log Age(yr) } \\
\hline & & & & & & & & & & & & & $S 00$ & $P 99$ & D97 \\
\hline C0718-218-00366 & 109.950218 & -21.908415 & 19.09 & & 1.77 & 1.06 & 2.07 & 14.48 & 0.61 & 0.07 & 1 & & 6.26 & 6.18 & \\
\hline С0718-218-00409 & 109.956973 & -21.893997 & 18.58 & & 1.25 & 0.74 & 1.49 & 16.11 & 0.33 & -0.69 & 1 & & 7.00 & 7.00 & \\
\hline С0718-218-00529 & 109.975359 & -21.915802 & 18.83 & & 1.52 & 0.92 & 1.85 & 14.67 & 0.67 & 0.05 & 1 & & 6.72 & 6.63 & 6.00 \\
\hline C0718-218-00608 & 109.988120 & -21.880320 & 18.67 & & 1.48 & 0.92 & 1.79 & 14.63 & 0.54 & 0.33 & 1 & & 6.68 & 6.60 & 6.00 \\
\hline C0718-218-00618 & 109.991959 & -21.989092 & 14.76 & 0.51 & 0.56 & 0.34 & 0.72 & 13.12 & 0.19 & -0.08 & 1 & & 6.85 & & \\
\hline C0718-218-00628 & 109.992614 & -21.905472 & 18.17 & & 1.57 & 0.96 & 1.86 & 13.90 & 0.70 & 0.19 & 1 & & 6.11 & 6.00 & \\
\hline C0718-218-00635 & 109.993885 & -21.946789 & 15.16 & 0.72 & 1.32 & 0.80 & 1.52 & 11.57 & 0.48 & 0.09 & 2 & 1.76 & 6.08 & & \\
\hline C0718-218-00647 & 109.995650 & -21.917452 & 17.75 & & 1.52 & 0.94 & 1.83 & 13.61 & 0.62 & 0.11 & 1 & & 6.08 & 6.00 & \\
\hline C0718-218-00656 & 109.998575 & -21.938709 & 15.75 & 0.84 & 1.49 & 0.90 & 1.74 & 11.65 & 0.57 & 0.02 & 2 & 2.32 & 6.08 & & \\
\hline C0718-218-00761 & 110.013962 & -21.901218 & 15.10 & 0.56 & 0.75 & 0.47 & 0.95 & 12.95 & 0.06 & 0.03 & 2 & 2.11 & 6.60 & & \\
\hline
\end{tabular}

in every cluster field. A total of 842 stars have been selected as PMS members in the different clusters, with respect to at least one of the three sets of isochrone models used to estimate PMS membership. For some stars, membership assignment and corresponding age values were obtained according to these three models.

For the first time, a catalogue of probable PMS cluster members has been elaborated. This catalogue is available at the CDS (Centre de Données astronomiques de Strasbourg), and it contains the basic information from the calculations and analysis described here. The column contents is as follows. Column 1: a 15character string with the star identification. The first 9 characters are the IAU cluster number, and the last five characters are the identification number in our photometric files. Columns 2 and 3 list the right ascension and declination in degrees for Epoch 2000; Columns 4-8 the optical photometric values $V,(U-B)$, $(B-V),(V-R)$, and $(V-I)$; Columns 9-11 the NIR photometric values $K,(J-H)$, and $(H-K)$; Column 12 the membership indication: 1, PMS member assignments in three CM diagrams; 2, PMS member assignments in all four CM diagrams. Column 13 indicates absorption $A_{V}=3.1 \times E(B-V)$ when available. Columns 14-16 show PMS ages with respect to the S00, P99, and D97 models, respectively. In Table 4 we list the first 10 lines of the catalogue as an example, which correspond to stars in NGC 2367.

The catalogue is meant as a starting point for more detailed studies of PMS stars, either individually or as members of hierarchically-structured stellar systems. In particular, we consider it as a basis for follow-up spectroscopic analysis and eventual observations in other wavelength ranges, mainly in the NIR range.

Acknowledgements. This work has been supported by the Spanish MCYT through grant AYA2004-05395 and by the Consejería de Educación y Ciencia de la Junta de Andalucía, through TIC 101. We made use of the NASA ADS Abstract Service and of the WEBDA data base, developed by Jean-Claude Mermilliod at the Laboratory of Astrophysics of the EPFL (Switzerland), and further developed and maintained by Ernst Paunzen at the Institute of Astronomy of the University of Vienna (Austria). This publication makes use of data products from the Two Micron All Sky Survey, which is a joint project of the University of Massachusetts and the Infrared Processing and Analysis Center/California Institute of Technology, funded by the National Aeronautics and Space Administration and the National Science Foundation.

\section{References}

Ahumada, A. V., Clariá, J. J., Bica, E., Dutra, C. M., \& Torres, M. C. 2001, A\&A, 377, 845
Alfaro, E. J., Cabrera-Caño, J., \& Delgado, A. J. 1991, ApJ, 378, 106 Alfaro, E. J., Cabrera-Caño, J., \& Delgado, A. J. 1992, ApJ, 399, 576 Baade, D., 1983, A\&AS, 51, 235

Balog, Z., \& Kenyon, S. 2002, AJ, 124, 2083

Balona L. A., \& Laney C. D. 1996, MNRAS, 281, 1341

Baume, G., Vázquez, R., Carraro, G., \& Feinstein, A. 2003, A\&A, 402, 549 [B03]

Baume, G., Vázquez, R. A., \& Feinstein, A. 1999, A\&AS, 137, 233

Bailyn, C., DePoy, D., Agostinho, R., et al. 1999, The First Year of Operations of the YALO Consortium, AAS, 195, 8706

Bessell, M. S., \& Brett, J. M. 1988, PASP, 100, 1134

Brandner, W., Grebel, E. K., Barbá, R. H., Walborn, N. R., \& Moneti, A. 2001, AJ, 122, 858

Carpenter, J. M. 2001, AJ, 121, 2851

Carraro, G., \& Patat, F. 2001, A\&A, 379, 136

Carraro, G., Patat, F., \& Baumgardt, H. 2001, A\&A, 107, 114

Claria, J. J. 1976, AJ, 81, 155

D’Antona, F., \& Mazzitelli, I. 1997, in Cool stars in Clusters and Associations, ed. R. Pallavicini, \& G. Micela, MemSAIt, 68, 807

DeGioia-Eastwood, K., Throop, H., Walker, G., \& Cudworth, K. M. 2001, ApJ, 549,578

Delgado, A. J., \& Alfaro, E. J. 2000, AJ, 119, 1848

Delgado, A. J., Alfaro, E. J., Moitinho, A., \& Franco, J. 1998, AJ, 116, 1801

Delgado, A. J., Miranda, L. F., \& Alfaro, E. J. 1999, AJ, 118, 1759

Delgado, A. J., Miranda, L. F., Fernández, M., \& Alfaro, E. J. 2004, AJ, 128, 330

Delgado, A. J., González-Martín, O., Alfaro, E. J., \& Yun, J. 2006, ApJ, 646, 269

de Winter, D., Koulis, C., Thé, P. S., et al. 1997, A\&AS, 121, 223

Feinstein, A., \& Marraco, H. G. 1980, PASP, 92, 266

Feinstein, A., Marraco, H. G., \& Forte, J. C. 1976, A\&AS, 24, 389

Fernandes, J., Lebreton, Y., \& Baglin, A. 1996, A\&A, 311, 127

FitzGerald, M. P., Kackson, P. D., Luiken, M., Grayzeck, E. J., \& Moffat, A. F. J. 1978, MNRAS, 182, 607

Flaccomio, E., Micela, G., \& Sciortino, S. 2006, A\&A, 455, 903

Forbes, D., \& DuPuy, D. 1978, AJ, 83, 266

Forte, J. C., \& Orsatti, A. M. 1984, ApJS, 56, 211

Girardi, L., Bertelli, G., Bressan, A., et al. 2002, A\&A, 391, 195

de Grijs, R., Gilmore, G. F., Mackey, A. D., et al. 2002, MNRAS, 337, 597

Guetter, H. H., \& Turner, D. G. 1997, AJ, 113, 2116

Hägele, G. F., Barbá, R. H., \& Bosch, G. L. 2003, BAAA, 46, 52

Haisch Jr., K. E., Lada, E. A., \& Lada, C. J. 2001, ApJ, 553, L153

Hartmann, L. 2005, in Star Formation in the Era of Three Great Observatories. Cambridge, MA

http: //cxc.harvard. edu/stars05/agenda/program.html

Herbig, G. H. 1998, ApJ, 497, 736

Herbst, W., \& Miller, D. P. 1982, AJ, 87, 1478

Hernández, J., Calvet, N., Hartmann, L., et al. 2005, AJ, 129, 856

Heske, A., \& Wendker, H., J. 1984, A\&AS, 57, 205

Hillenbrand, L. A., Massey, P., Strom, S. E., \& Merrill, K. M. 1993, AJ, 106, 1906

Hillenbrand, L. A., \& Hartmann, L. W. 1998, ApJ, 492, 540

Jeffries, R. D., \& Oliveira, J. M. 2005, MNRAS, 358, 13

Kharchenko, N. V., Piskunov, A. E., Röser, S., Schilbach, E., \& Scholz, R.-D. 2005, A\&A, 438, 1163 
Kenyon, S. J., \& Hartmann, L. 1995, ApJS, 101,117

Lada, E. A. 2005, Cores to Clusters: Star Formation with Next Generation Telescopes, 141

Lada, E. A., \& Alves, J. F. 2004, IAU Symp., 221, 3

Lawson, W. A., Lyo, A-R., \& Muzzerolle, J. 2004, MNRAS, 351, L39

Luhman, K. L., Stauffer, J. R., Muench, A. A., et al. 2003, ApJ, 593, 1093

Lyo, A-R., Lawson, W. A., \& Bessell, M. S. 2004, MNRAS, 355, 363

Mamajek, E. E., Meyer, M. R., \& Liebert, J. 2002, AJ, 124, 1670

Marco, A., \& Negueruela, I. 2002, A\&A, 393,195

Marco, A., Bernabeu, G., \& Negueruela, I. 2001, AJ, 121,2075

Marschall, L. A., Comins, N. F., \& Karshner, G. B. 1990, AJ, 99, 1536

Massey, P., DeGioia-Eastwood, K., \& Waterhouse, E. 2001, AJ, 121, 1050

Moffat, A. F. J., \& Vogt, N. 1973, A\&AS, 10, 135

Moffat, A. F. J., \& Vogt, N. 1975, A\&AS, 20, 125

Munari, U., Carraro, G., \& Barbon, R. 1998, MNRAS, 297, 867

Palla, F., \& Staller, S. W. 1999, ApJ, 525, 772

Palla, F., Randich, S., Flaccomio, E., \& Pallavicini, R. 2005, ApJ, 626, L49

Park, B.-G., \& Sung, H. 2002, AJ, 123, 892

Park, B.-G., Sung, H., Bessell, M. S., \& Kang, Y. H. 2000, AJ, 120, 894

Patat, F., \& Carro, G. 2001, MNRAS, 325, 1591

Pozzo, M., Naylor, T., Jeffries, R. D., \& Drew, J. E. 2003, MNRAS, 341, 805

Prisinzano, L., Damiani, F., Micela, G., \& Sciortino, S. 2005, A\&A, 430, 941

Raboud, D., Cramer, N., \& Bernasconi, P. A. 1997, A\&A, 325, 167

Raboud, D., \& Mermilliod, J. C., 1998, A\&A, 333, 897

Sagar, R., \& Joshi, U. C. 1979, Ap\&SS, 66, 3

Sagar, R., \& Cannon, R. D. 1997, A\&AS, 122, 9

Sagar, R., \& Joshi, U. C. 1981, Ap\&SS, 75, 465

Sanner, J., Brunzendorf, J., Will, J.-M., \& Geffert, M. 2001, A\&A, 369, 511

Sartori, M. J., Lépine, J. R. D., \& Dias, W. S. 2003, A\&A, 404, 913
Sicilia-Aguilar, A., Hartmann, L. W., Hernández, J., Briceño, C., \& Calvet, N. 2005, AJ, 130, 188

Shobbrook, R. R. 1983, MNRAS, 205, 1215

Siess, L., Dufour, E., \& Forestini, M. 2000, A\&A, 358, 593

Slesnick, L. S., Hillenbrand, L. A., \& Massey, P. 2002, ApJ, 576, 880

Stauffer, J., Hartmann, W. L., Jones, B. F., \& McNamara, B. R. 1989, ApJ, 342, 285

Stone, R. C. 1988 , AJ, 96, 1389

Sung, H., Bessell, M. S., \& Lee, S.-W. 1997, AJ, 114, 2644

Sung, H., Bessell, M. S., \& Lee, S.-W. 1998, AJ, 115, 734

Sung, H., Chun, M.-Y., \& Bessell, M. S. 2000, AJ, 120, 333

Thé, P. S., Hageman, T., Westerlund, B. E., \& Tjin A Djie, H. R. E. 1985, A\&A, 151,391

Trullols, E., \& Jordi, C. 1997, A\&A, 324, 549

Turner, D. G. 1994, RMAA, 29, 163

Turner, D. G., Grieve, G. R., Herbst, W., \& Harris, W. E. 1980, AJ, 85, 1193

Turner, D. G., \& Moffat, A. F. J. 1980, MNRAS, 192, 283

van den Ancker, M. E., Thé, P. S., Feinstein, A., et al. 1997, A\&AS, 123, 63

van den Ancker, M. E., Thé, P. S., \& de Winter, D. 2000, A\&A, 362, 580

Vázquez, R. A., \& Feinstein, A. 1990, A\&AS, 86, 209

Vázquez, R. A., \& Feinstein, A. 1991, A\&AS, 87, 383

Vázquez, R. A., Baume, G., Feinstein, A., \& Prado, P. 1994, A\&AS, 106, 339

Veschueren, W., Hensberge, H., \& De Loore, C. 1990, Ap\&SS, 170, 245

Vogt, N., \& Moffat, A. F. J. 1972, A\&AS, 7, 133

Walker, M. F. 1961, ApJ, 133, 43

Wesselink, A. J. 1969, MNRAS, 146, 329

Witt, A. N., Schild, R. E., \& Kraiman, J. B. 1984, ApJ, 281, 708 